\title{
Use of a Self-Delivering Anti-CCL3 FANA Oligonucleotide as an Innovative Approach to Target Inflammation after Spinal Cord Injury
}

\author{
Nicolas Pelisch, ${ }^{1,3}$ Jose Rosas Almanza, ${ }^{1,3}$ Kyle E. Stehlik, ${ }^{1,3}$ Brandy V. Aperi, ${ }^{1,3}$ and Antje Kroner ${ }^{1,2,3}$ \\ https://doi.org/10.1523/ENEURO.0338-20.2021 \\ ${ }^{1}$ Department of Neurosurgery, Medical College of Wisconsin, Milwaukee, WI 53226, ${ }^{2}$ Department of Microbiology and \\ Immunology, Medical College of Wisconsin, Milwaukee, WI 53226, and ${ }^{3}$ Clement J. Zablocki Veterans Affairs Medical \\ Center, Milwaukee, WI 53295
}

\begin{abstract}
Secondary damage after spinal cord injury (SCl) occurs because of a sequence of events after the initial injury, including exacerbated inflammation that contributes to increased lesion size and poor locomotor recovery. Thus, mitigating secondary damage is critical to preserve neural tissue and improve neurologic outcome. In this work, we examined the therapeutic potential of a novel antisense oligonucleotide (ASO) with special chemical modifications [2'-deoxy-2-fluoro-D-arabinonucleic acid (FANA) ASO] for specifically inhibiting an inflammatory molecule in the injured spinal cord. The chemokine CCL3 plays a complex role in the activation and attraction of immune cells and is upregulated in the injured tissue after SCI. We used specific FANA ASO to inhibit CCL3 in a contusive mouse model of murine SCI. Our results show that self-delivering FANA ASO molecules targeting the chemokine CCL3 penetrate the spinal cord lesion site and suppress the expression of CCL3 transcripts. Furthermore, they reduce other proinflammatory cytokines such as tumor necrosis factor (TNF) and interleukin (IL)-1 $\beta$ after SCI. In summary, we demonstrate for the first time the potential of FANA ASO molecules to penetrate the spinal cord lesion site to specifically inhibit CCL3, reducing proinflammatory cytokines and improve functional recovery after SCl. This novel approach may be used in new treatment strategies for $\mathrm{SCl}$ and other pathologic conditions of the CNS.
\end{abstract}

Key words: CCL3; FANA ASO; inflammation; novel RNA inhibitor; secondary damage; spinal cord injury

\section{Significance Statement}

Preserving white matter tissue following spinal cord injury (SCl) is an important therapeutic goal to minimize tissue damage and improve neurologic outcome. Unresolved inflammation contributes to secondary tissue damage. Specifically targeting proinflammatory molecules, such as the chemokine CCL3, in the injured spinal cord can present technical challenges including tissue penetration, stability and efficacy of suppression. Here, we present the use of novel 2'-deoxy-2-fluoro-D-arabinonucleic acid (FANA) antisense oligonucleotide (ASO) molecules as a feasible approach to specifically target proinflammatory molecules in the injured spinal cord.

\section{Introduction}

Spinal cord injury (SCl) causes severe clinical and socioeconomic problems in affected individuals who often face a multifaceted spectrum of complex long-term

\footnotetext{
Received August 4, 2020; accepted January 1, 2021; First published February 25, 2021.

The authors declare no competing financial interests.
}

sequelae, including loss of motor and sensory function and impairment of autonomic nervous system function (Singh et al., 2014). Despite considerable progress in primary and rehabilitative care, curative therapies

Author contributions: N.P., J.R.A., and A.K. designed research; N.P., J.R.A., K.E.S., B.V.A., and A.K. performed research; N.P., J.R.A., K.E.S., and A.K. analyzed data; N.P., J.R.A., and A.K. wrote the paper. 
remain elusive, resulting in $\mathrm{SCl}$ remaining a significant cause of disability and mortality (Sekhon and Fehlings, 2001). Tissue damage after $\mathrm{SCl}$ is first categorized as primary damage caused by the initial trauma that triggers subsequent pathophysiological cascades and cellular changes classified as secondary tissue damage. Excessive and unresolved inflammation after $\mathrm{SCl}$ can contribute to the secondary tissue damage and thus enhance functional impairment. However, the inflammatory response to injury is essential for the clearance of cellular debris and tissue remodeling (Blight, 1994; Donnelly and Popovich, 2008). Factors regulating this inflammatory response after $\mathrm{SCl}$, like the chemokine-ligand/receptor-system, are of particular interest. CCL3, also known as macrophage inflammatory protein (MIP)$1 \alpha$, is a member of the C-C-subfamily of low molecular weight chemokines. This chemokine is transcriptionally regulated during inflammation (Banisor et al., 2005). It is detectable in most mature hematopoietic cells including monocytes, macrophages (Standiford et al., 1993) and neutrophils (Kasama et al., 1993), as well as microglia and astrocytes (Peterson et al., 1997; Sun et al., 1997). CCL3 is known to play a complex role in the activation and recruitment of immune cells and has been reported to be upregulated after $\mathrm{SCl}$ (Bartholdi and Schwab, 1997; Lee et al., 2000; Francos-Quijorna et al., 2017; Zhang and Yang, 2017). Using $C C L 3^{-1-}$ mice, we have previously shown that the absence of CCL3 results in reduced lesion volume and improved functional recovery. Furthermore, we demonstrated a marked reduction of other proinflammatory cytokines like tumor necrosis factor (TNF) and interleukin (IL)-1 $\beta$ (Pelisch et al., 2020). Based on these findings, we concluded that CCL3 may be a promising target for reducing the inflammatory response and secondary damage after SCl. The goal of this project was to specifically target CCL3 in a therapeutically feasible way.

Modulating inflammation inside the injured spinal cord can be challenging, specifically targeting single molecules. Specific inactivation of mRNA can be a valuable approach, but some technical difficulties pose serious challenges. Antisense oligonucleotides (ASOs) are single-stranded, synthetic nucleic acids that recognize and specifically bind mRNA via Watson-Crick base pairing (Bennett and Swayze, 2010). Binding results in degradation of target mRNA by RNase $\mathrm{H}$-dependent mechanisms or prevention of translation or splicing. Although ASO's potential was established

This work was supported by the Wings for Life Spinal Cord Research Foundation Grant WFL-US-13/16; by a pilot Grant from the Department of Neurosurgery, Medical College of Wisconsin; and startup funding from Advancing a Healthier Wisconsin Endowment (AHW).

Acknowledgements: We thank Dr. Kajana Satkunendrarajah and Dr. Karin Swartz for helpful discussions as well as Dena Hall and the VMU staff for excellent animal care; Christy Stadig, Stephanie Flaherty, and Sara Jackson for administrative support, and Veenu Aishwarya, AUM LifeTech, Inc., for valuable discussions and technical support.

Correspondence should be addressed to Antje Kroner at akroner@mcw.edu.

https://doi.org/10.1523/ENEURO.0338-20.2021 Copyright $@ 2021$ Pelisch et al.

This is an open-access article distributed under the terms of the Creative Commons Attribution 4.0 International license, which permits unrestricted use, distribution and reproduction in any medium provided that the original work is properly attributed. decades ago, actual development of ASO-based drugs faced major challenges (Bhagavati, 2016; Chi et al., 2017; Gustincich et al., 2017). The therapeutic application of ASOs was found to be limited by their high susceptibility to degradation by endogenous nucleases and poor bioavailability. Additionally, ASOs may struggle to cross vascular endothelium, dense extracellular matrix and cell and nuclear membranes to reach their intracellular targets. Thus, introduction of chemical modifications that improve these limitations has become an essential feature in designing ASOs. A variety of sugar modifications contribute to resistance against nucleases and improve binding affinity. 2'-deoxy-2-fluoro-D-arabinonucleic acid (FANA) is an oligonucleotide analog comprised of $2^{\prime}$-deoxy-2'-fluoroarabinonucleotides, which, unlike many $2^{\prime}$-modified analogs that show RNA-like properties, is considered a DNA mimic (Martín-Pintado et al., 2012), forming FANA:RNA hybrids that mirror the structure of the native DNA:RNA hybrid (Denisov et al., 2001). As a result, FANA, and particularly chimeric FANA-DNA ASOs, are able to elicit RNase $\mathrm{H}$-mediated cleavage of target RNA (Lok et al., 2002; Min et al., 2002). Another unique property of FANA ASO molecules is their capability of gymnotic delivery in vitro and in vivo. Smaldone et al. (2019) demonstrated a $\sim 99 \%$ transfection efficiency using FANA ASO technology in a leukemia cell line.

FANA ASO molecules targeting a variety of genes have been used in various models. For instance, FANA ASOs targeting the HIV-1 genome successfully suppressed viral replication in $\mathrm{CD}^{+}{ }^{+} \mathrm{T}$-cells in culture (Takahashi et al., 2019). In other work, FANA ASO molecules were used to confirm a direct link between Abi-1 loss and cell cycle activity in hematopoietic stem cells. Abi-1 protein levels in healthy $\mathrm{CD}_{3}{ }^{+}$cells that were incubated with FANAABI1-ASO were downregulated $>50 \%$ compared with scramble control (Chorzalska et al., 2018). In vivo, FANA ASO mediated inhibition of phosphodiesterase-4 and phosphodiesterase-7 reduced smoke-induced lung inflammation in mice when administered intratracheally (Fortin et al., 2009). Although the efficacy of ASO in degenerative brain disorders such as Huntington's disease has been demonstrated (Kordasiewicz et al., 2012; Østergaard et al., 2013), the therapeutic potential of FANA ASOs has never been evaluated for SCl. The present study, to our knowledge, is the first to evaluate anti-inflammatory efficacy of FANA ASOs in the murine spinal cord, using a mouse $\mathrm{SCl}$ model of thoracic contusion $\mathrm{SCl}$. Here, we demonstrate the potential of FANA ASO molecules targeting CCL3 to penetrate the spinal cord lesion site, suppress the expression levels of CCL3, reduce levels of proinflammatory cytokines and improve functional recovery after $\mathrm{SCl}$. This novel approach may result in new treatment strategies not only for SCI but also other pathologic conditions of the CNS.

\section{Materials and Methods}

\section{FANA ASO and administration}

Four different FANA ASOs targeting CCL3 used in this study were designed and synthesized by AUM BioTech, LLC based on the CCL3 mRNA sequence obtained from 
NCBI (NM_011337.2) as follows: CCL3\#1: AATAGTCA ACGATGAATTGGC; CCL3\#2: GACTCTCAGGCATTCAG TTCC; CCL3\#3: TCAAGTGAAGAGTCCCTCGAT; CCL3\#4: CTACCTAGAATAGCTGTCACC. Scrambled FANA ASO was used as a negative control. Where indicated, FANA ASO with a Cy3 (in vitro and histology) or FAM (flow cytometry) fluorescent tags were used.

\section{Spinal cord contusion injury and FANA ASO treatment}

All procedures were approved by and performed in accordance with the Institutional Care and Use Committees of the Clement J. Zablocki Veterans Affairs Medical Center and the Medical College of Wisconsin. Young adult 8- to 10-week-old female wild-type C57BL/6 mice (18-22 g) were obtained from Charles River Laboratories and used for all experiments. For SCl, mice were deeply anesthetized with isoflurane (4\% induction, $2.5 \%$ maintenance), and a moderate contusion was induced at the T11 thoracic level using the Infinite Horizon Impactor device (Precision Systems and Instrumentation, LLC) with a contusion force of $40 \mathrm{kdyn}$. Control animals were subjected to a laminectomy without contusion. Animals received subcutaneous carprofen $(5 \mathrm{mg} / \mathrm{kg})$ twice daily for $3 \mathrm{~d}$ postinjury. Bladders were expressed twice daily until micturition reflexes returned.

Intrathecal injection was performed as previously described (Njoo et al., 2014) at different time points indicated for the individual experiments. Briefly, mice were anesthetized with isoflurane as described above and a lumbar puncture was performed between the groove of lumbar vertebrae L5 and L6 to deliver anti-CCL3 FANA ASO or a scrambled control using a 30-G needle. A tail flick was used as a confirmation that the needle entered the intradural space and $10 \mu \mathrm{l}$ of solution $(10 \mathrm{mg} / \mathrm{kg})$ was slowly administered. The treatment paradigm consisted of one to three doses, with the first dose given immediately after the injury and additional doses every $24 \mathrm{~h}$, where indicated. In one cohort, pretreatment was performed $24 \mathrm{~h}$ before injury, followed by another dose immediately after injury and the last dose $24 \mathrm{~h}$ later.

\section{Locomotor assessment}

The Basso mouse scale (BMS) open-field locomotor test with a score range of 0-9 (Basso et al., 2006) was used to assess functional recovery during a period of 28 to $35 \mathrm{~d}$. Mice ( $n=10-13$ per group) were evaluated for locomotor function at days $1,3,7,10$, and 14 postinjury and weekly thereafter by two individuals blinded to experimental conditions and trained in BMS analysis by Basso's laboratory at Ohio State University. Consensus scores for each animal were averaged at each time point for a maximum of nine points for the BMS score and 11 points for the sub score, which assesses finer aspects of locomotion. This locomotor assessment was performed in two separate cohorts of mice.

\section{Tissue collection}

For tissue collection, female wild-type mice were euthanized with an overdose of phenytoin/pentobarbital
$(120 \mathrm{mg} / \mathrm{kg})$ at $1,3,7 \mathrm{~d}$ after injury for mRNA analysis $(n=$ 5-6 mice per time point). After transcardial perfusion with ice-cold, fresh $1 \times$ PBS, a 5-mm piece of spinal cord centering on the lesion was dissected out, and immediately snap frozen. RNA was extracted using the RNeasy Lipid Tissue Mini kit (QIAGEN) according to the manufacturer's instructions, followed by RNA quantification and characterization of purity using Nanodrop 2000 spectrophotometer (Thermo Scientific) and reverse transcribed for $1 \mu \mathrm{g}$ of RNA using QuantiTect Reverse Transcription kit (QIAGEN).

\section{Bone marrow-derived macrophages (BMDMs)}

BMDMs were generated as previously described (Longbrake et al., 2007) from adult female C57/BL6 mice. Mice were euthanized and femurs removed. Bone marrow was flushed out, homogenized and red blood cells were hypotonically lysed. After washing, cells were cultured in RPMI media containing $20 \%$ fetal bovine serum (FBS), 1\% penicillin/streptomycin, 1\% vitamins (all from Invitrogen), and 10\% L-cell-conditioned media (LCCM) as a source of M-CSF for $7 d$ until cells matured into adherent macrophages. BMDMs were harvested with $0.25 \%$ trypsin, re-plated at $1 \times 10^{6}$ cells/ well and cultures were stimulated with lipopolysaccharide (LPS; $100 \mathrm{ng} / \mathrm{ml}$, Sigma, L2654) as previously described (Kroner et al., 2014), followed by $10 \mu \mathrm{mol}$ FANA ASO or scrambled control treatment for $24 \mathrm{~h}$. After stimulation, cells were lysed in RLT buffer (QIAGEN) and mRNA was extracted using the RNeasy Mini kit (QIAGEN) followed by RNA quantification and reverse transcription as described above.

\section{Q-PCR}

Q-PCR was performed in duplicates using a PCR thermal cycler (LightCycler 480 System, Roche) with specific primers using LightCycler 480 Mastermix (Roche). Gene expression levels were analyzed using the $\Delta \Delta$ ct method normalized to peptidylprolyl isomerase $A$ (PPIA) as a housekeeping gene and laminectomy as a baseline. Primer sequences for the genes analyzed are as follows: PPIA: ATG TGC CAG GGT GGT GAC TTा A (forward primer $5^{\prime}-3^{\prime}$ ); TGT GTT TGG TCC AGC ATT TGC C (reverse primer $\left.5^{\prime}-3^{\prime}\right)$, CCL3: CAG CTT ATA GGA GAT GGA GCT ATG (forward primer 5'-3'); TCA CTG ACC TGG AAC TGA ATG (reverse primer 5'-3'), TNF: TTG CTC TGT GAA GGG AAT GG (forward primer 5'-3'); GGC TCT GAG GAG TAG ACA ATA AAG (reverse primer $5^{\prime}-3^{\prime}$ ), IL-1 $\beta$ : ATG GGC AAC CAC TTA CCT ATT T (forward primer $5^{\prime}-$ 3'); GTT CTA GAG AGT GCT GCC TAA TG (reverse primer $\left.5^{\prime}-3^{\prime}\right)$.

\section{ELISA}

Tissue was harvested as described above at days 1, 3, and 7 after $\mathrm{SCl}$ ( $n=5$ per time point) and snap frozen. Later, tissue was homogenized as previously described (Sato et al., 2012), and CCL3 and TNF protein concentration was quantified in duplicates using the ELISA kit 
(CCL3: MMA00, R\&D Systems; TNF: Invitrogen, \#887324).

\section{Flow cytometry}

Flow cytometry was performed $7 \mathrm{~d}$ after contusion injury and intrathecal injection of FAM fluorescently labeled FANA ASO molecules as described above to detect the uptake of FANA ASO molecules. Naïve, uninjured mice served as controls. In an additional experiment, flow cytometry was done $1 \mathrm{~d}$ after $\mathrm{SCl}$ to detect the number of neutrophils in the injured tissue after intrathecal injection of unlabeled CCL3-FANA ASO or scrambled control molecules. Mice were transcardially perfused with PBS, and a $5-\mathrm{mm}$ section of the spinal cord containing the lesion site was dissected Cells were extracted using the Adult Brain Dissociation kit (Miltenyi, \#130-107-677) according to the manufacturer's instructions. Flow cytometry staining was performed following standard protocols as previously described (Kroner et al., 2014). Nonspecific antibody binding was blocked by incubation with $5 \%$ bovine serum albumin (BSA), followed by staining with antibodies against CD11b (1:500, 17-4031-82, clone M1/70, rat anti-mouse, APC, Thermo Fisher Scientific) and CD45 (1:500, 25-0451-82, clone 30-F11, rat anti-mouse, PE-Cy7, Thermo Fisher Scientific). Cells from animals at $1 \mathrm{~d}$ after $\mathrm{SCl}$ without fluorescent FANA ASO injection were additionally stained with anti-Ly6G/C (Gr-1; 1:50, 11-5931-82, Clone RB6-8C5, rat anti mouse, FITC, Invitrogen). Compensation was performed using a compensation particle set (BD Biosciences, \#552844). Cells were analyzed using a BD FACS Aria Fusion flow cytometer with FACS Diva software. Results were analyzed using FlowJo software.

\section{Histology and immunofluorescence}

At 5, 7, or $28 d$ after contusion injury and intrathecal injection with FANA ASO molecules as described above (Cy3 labeled for day 7 , unlabeled for days 5 and 28), mice were euthanized as described above, and transcardially perfused with $1 \times$ PBS, followed by perfusion fixation with cold $4 \%$ paraformaldehyde (PFA); 1-cm-long sections of the spinal cord centering on the lesion site were dissected and postfixed in 4\% PFA for $2 \mathrm{~h}$, and cryoprotected in 30\% sucrose solution. The cords were then embedded in OCT compound (Sakura Finetek) and serial cross sections $(14 \mu \mathrm{m})$ were cut and picked up on glass slides (Superfrost Plus Gold; Fisher Scientific). Immunofluorescence labeling was performed as follows: nonspecific binding was blocked by $3 \mathrm{~h}$ of incubation in 2\% BSA, $0.1 \%$ Triton X-100 and $5 \%$ normal goat serum. Sections were then incubated overnight at $4^{\circ} \mathrm{C}$ with the following antibodies: glial fibrillary acidic protein (GFAP) for astrocytes (1:800, Z0334, rabbit anti-mouse; DAKO), CD11b (1:200, MCA711G, rat anti-mouse, Bio-Rad), Neurofilament SMI-32P (1:1500, 801702, mouse antirabbit; BioLegend), and NeuN (1:100, MAB377, mouse anti-rabbit, Millipore Sigma). For primary antibodies produced in mouse, $1 \%$ of fragment goat anti-mouse lgG (1:22, 115-007-003, Jackson ImmunoResearch) was added to the blocking solution. FluoroMyelin Red for myelin content (1:150; F34652 Thermo Fisher Scientific) was co-stained with GFAP antibody as previously described (Brennan et al., 2016). Slides were then incubated with secondary antibodies (1:400, Alexa Fluor 488 goat-anti-mouse lgG, A11029 and Alexa Fluor 647 goat anti-rabbit; A21245, both Thermo Fisher Scientific) for $1 \mathrm{~h}$ at room temperature, followed by washing steps and mounting with DAPI Nector Labs \#6149856).

\section{Image analysis and quantification}

Images were captured using a Nikon Eclipse E600 equipped with a digital camera or a Leica TCS SP8 Laser Scanning Confocal Microscope. Analyses of histologic sections were performed using ImageJ (ImageJ-win64 2.0). The epicenter of injury in all spinal cords was identified, followed by quantification of myelin loss using Fluoromyelin-stained cross sections of the spinal cord. Areas devoid of GFAP labeling were measured at $112-\mu \mathrm{m}$ intervals by calculating the GFAP-devoid area as a percentage of the total area of the cross section.

For the quantification of astrocytes, macrophages $/ \mathrm{mi}$ croglia and SMI-32 + profiles, a region of interest covering a $290 \times 290 \mu \mathrm{m}$ area was captured laterally perilesional to the lesion core at the epicenter site with $14 \mathrm{z}$-stack sections $(1 \mu \mathrm{m})$ spanning the complete axial thickness $(14 \mu \mathrm{m})$.

DAPI nuclei of astrocytes, microglia/macrophages were manually quantified through a continuous viewing of all zstack sections. This allowed for greater resolution and ability to distinguish individual cells in presence of complex ramified cellular morphologies following damage progression. SMI-32+ endbulbs were quantified following a similar approach. All analyses were done by a blinded investigator.

\section{Statistical analyses}

Comparisons between two datasets were analyzed by Student's $t$ test. Data with more than two variables were analyzed by ordinary one-way ANOVA (different time points in different samples) two-way repeated measures ANOVA (different time points in the same samples) with post hoc Tukey's or Dunnett's analysis. $\chi^{2}$ test was used for testing relationships categories (GraphPad Prism version 8). All ps $<0.05$ were considered statistically significant.

We also applied estimation based on confidence intervals $(\mathrm{Cls})$. For this, we directly uploaded raw data in https://www.estimationstats.com/ and included the results (Ho et al., 2019). The mean difference for two comparisons is reported. Five thousand bootstrap samples were taken; the $\mathrm{Cl}$ was bias-corrected and accelerated. To measure the effect size, we included unbiased Cohen's $d$ (standardized mean difference).

\section{Results}

\section{FANA ASO molecules targeting CCL3 penetrated BMDMs in a gymnotic fashion and reduced CCL3 expression in vitro}

To our knowledge, this is the first study using CCL3 targeting FANA ASO molecules. We first investigated 

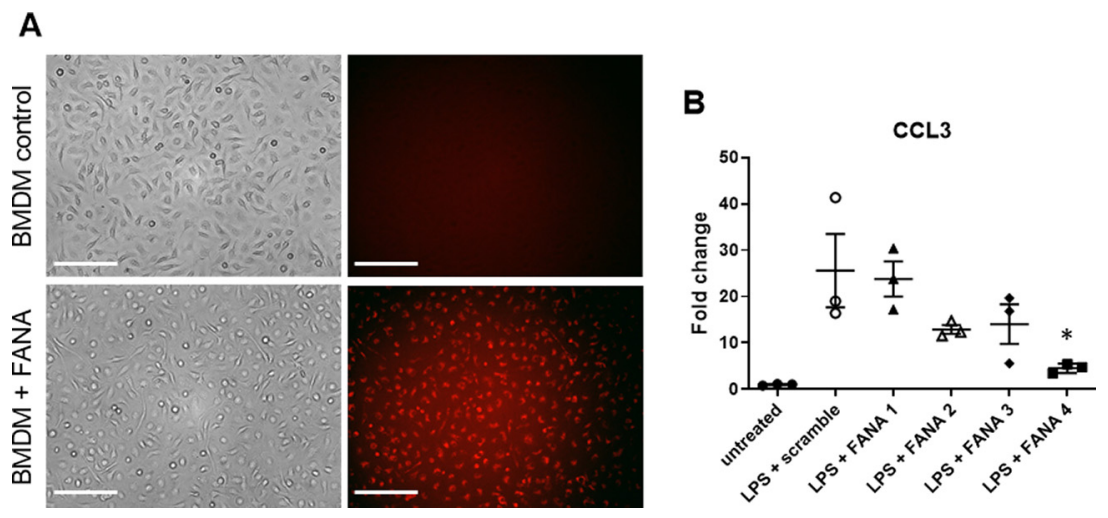

Figure 1. FANA ASO molecules penetrate BMDMs in a gymnotic fashion and reduce CCL3 expression in vitro. $\boldsymbol{A}$, When added to the tissue culture media, fluorescently labeled FANA ASO molecules penetrate BMDM cells after $2 \mathrm{~h}$ of incubation (red fluorescence, lower panel). The upper panel demonstrates the absence of fluorescence when no FANA ASO molecules were added to the media. Scale bar: $50 \mu \mathrm{m}$. B. Assessment of CCL3 knock-down in LPS stimulated BMDMs using four different FANA ASOs molecules. CCL3 mRNA expression levels were compared between LPS-treated BMDMs in the presence of scrambled control FANA ASO or the individual anti-CCL3 FANA ASOs. CCL3 expression levels were unchanged in the presence of FANA-CCL3\#1, FANA-CCL3\#2, and FANA-CCL3\#3. Treatment with FANA-CCL3\#4 resulted in a significant reduction of CCL3 expression by $\sim 80 \%$. Untreated BMDMs are included for reference; $n=3$ /group; ${ }^{*} p<0.05$. Error bars are represented as SEM.

whether fluorescently labeled FANA ASO molecules were able to penetrate BMDMs in a gymnotic fashion. For this, FANA ASO molecules were added directly to the cell culture media at a final concentration of $10 \mu \mathrm{m}$. After $2 \mathrm{~h}$ of incubation, all cells showed red fluorescence, indicating FANA ASOs had penetrated them compared with media without FANA ASOs (Fig. 1A). Next, we assessed the potential of CCL3 knock-down in LPS stimulated BMDMs using four different FANA ASO molecules. BMDMs were stimulated with LPS to increase CCL3 expression, which is very low in unstimulated cells. CCL3 mRNA expression levels were compared between LPS-treated BMDMs in the presence of scrambled control FANA ASOs and the individual anti-CCL3 FANA ASO. CCL3 expression levels were not significantly changed in the presence of FANACCL3\#1, FANA-CCL3\#2, and FANA-CCL3\#3. However, treatment with FANA-CCL3\#4 resulted in a significant reduction of CCL3 expression by $\sim 80 \%$ (Fig. 1B). Accordingly, all following inhibition experiments were performed using FANACCL3\#4.

\section{Fluorescently labeled FANA ASOs could be detected at the $\mathrm{SCl}$ lesion site after intrathecal injection}

Next, we assessed the feasibility of intrathecal delivery of FANA ASO molecules. The rationale for intrathecal administration was to reduce systemic exposure and reduce anti-inflammatory effects in the periphery. To assess whether FANA ASOs were reaching the lesion site, we injected $10 \mathrm{mg} / \mathrm{kg}$ of fluorescently labeled FANA ASOs intrathecally in $\mathrm{SCl}$ and naive, uninjured mice. Seven days after injection, we verified the presence of FANA ASO at the lesion site (Fig. 2). Using flow cytometry, we could demonstrate the presence of FANA ASO molecules in CD11b+/CD45low microglia (MG) and CD11b+/CD45high monocyte-derived macrophages (MDMs; Fig. 2A). The percentage of labeled cells showed wide variability, possibly indicative of less successful injections.
The percentage of labeled microglia was similar between naive and injured mice while very low MDMs were detected in naive mice (Fig. 2B). FANA ASO labeling at the lesion site was also assessed histologically. The presence of red (Су3)-fluorescent labeling, an indication of FANA ASO uptake, was observed in CD11b+ macrophages/microglia and GFAP+ astrocytes, demonstrating that FANA ASOs are not taken up by any specific cell type. However, no FANA ASO uptake was detected in $\mathrm{NeuN}+$ neurons (Fig. 2C).

\section{The inflammatory response following $\mathrm{SCl}$ was reduced after FANA ASO injection}

After establishing that FANA ASOs penetrated the lesion site after lumbar intrathecal injection, we assessed the efficacy of suppressing CCL3 expression at different time points after injury. A single injection of FANA ASO CCL3\#4 resulted in significant suppression of CCL3 mRNA in the injured spinal cord compared with injection of scrambled control at day 3 postinjury. This reduction was accompanied by the suppression of TNF and IL-1 $\beta$ transcripts, indicating a reduction of the proinflammatory tissue environment. However, this effect was not sustained beyond day 3 (Fig. $3 A$ ). To achieve a longer lasting suppression of CCL3, we used a two-injection paradigm immediately after surgery and $24 \mathrm{~h}$ postinjury, which confirmed CCL3, TNF, and IL-1 $\beta$ suppression only at day 3 (Fig. 3B). However, when three injections were administered every $24 \mathrm{~h}$ for $3 \mathrm{~d}$, sustained suppression of CCL3 was detected at day 7 , reducing CCL3 levels by $\sim 60 \%$. Both TNF and IL-1 $\beta$ expression levels were also reduced by $\sim 50 \%$ (Fig. $3 C$ ). Suppression of CCL3 in the injured spinal cord was confirmed at the protein level using ELISA at 1,3 , and $7 \mathrm{~d}$ after $\mathrm{SCl}$. TNF protein suppression was sustained at days 1 and 3 . At day 7 , overall TNF expression had returned to baseline expression levels and was not different between CCL3 FANA ASO and controltreated mice (Fig. 3D). 


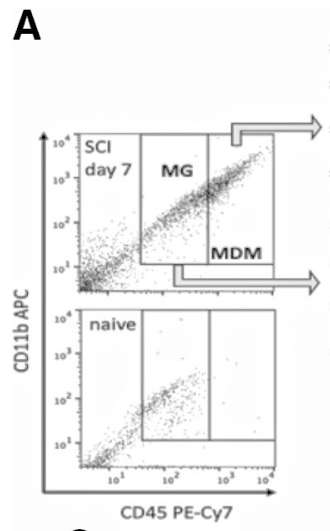

C
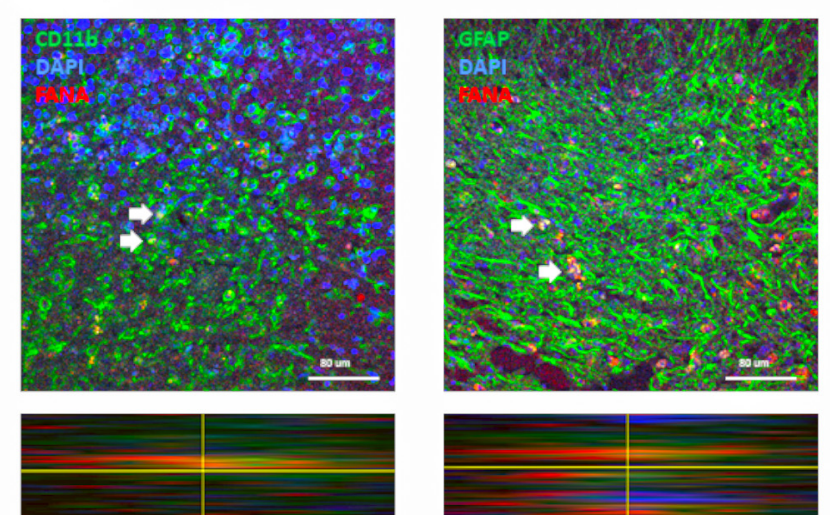

Figure 2. Fluorescently labeled FANA ASOs are present at the SCI lesion site after intrathecal injection. $\boldsymbol{A}$, Representative images of flow cytometry on cells from a section of spinal cord tissue centering on the lesion or spinal cord tissue from naive mice. Green fluorescence derived from FAM-labeled FANA was detectable in gated cell populations for CD11b+/CD45low microglia (MG) and CD11b+/CD45high MDMs. MDM are not detected in naive tissue. B, Quantification of percentages of MG and MDM containing FAM-labeled FANA; $n=4$ /group, error bars are represented as SEM. $\boldsymbol{C}$, Seven days after SCI and intrathecal injection, labeled FANA ASO was detectable at the lesion site (red staining). Confocal images with orthogonal view demonstrate uptake of FANA molecules in a non- cell specific manner, as shown by the presence in CD11b + macrophages/microglia and GFAP+ astrocytes. No uptake was detected in NeuN+ neurons; $n=4-5 /$ group. Scale bars: $80 \mu \mathrm{m}$.

\section{Treatment with anti-CCL3 FANA ASOs resulted in mild functional improvement}

We then assessed the therapeutic potential of CCL3 inhibition using FANA ASO. To this end, we induced a moderate contusion injury at T11, followed immediately by intrathecal injection of CCL3-FANA ASO or scrambled control, and repeated injections after 24 and $48 \mathrm{~h}$. BMS scores in this cohort did not show significant improvement compared with control animals (Fig. 4A). However, a significantly higher percentage of CCL3FANA ASO-treated mice performed plantar placement on days 5-7 after injury, compared with control animals (Fig. 4B). To ensure sufficient time for CCL3 knockdown, we also investigated the efficacy of CCL3-FANA ASO pretreatment, with the first dose given $24 \mathrm{~h}$ before injury, followed by two more injections immediately after injury and after $24 \mathrm{~h}$. BMS scores were also not significantly different in treated versus control animals (Fig. 4C), but the pretreatment group showed a significant increase of plantar placement at days 3 through 7 after injury compared with controls (Fig. 4D), indicating that CCL3 inhibition with FANA ASOs may result in a mild functional improvement.

\section{Treatment with anti-CCL3 FANA ASOs reduced CD11b + macrophages at the lesion site}

Next, we assessed whether treatment with CCL3-FANA ASO influenced the cellular composition at the lesion site. Gr-1 + cells were quantified using flow cytometry $1 \mathrm{~d}$ after $\mathrm{SCl}$. No difference in neutrophil numbers was detected between the groups $\left(M_{\text {diff }}=-11.6[95.0 \% \mathrm{Cl}-36.8\right.$, 2.45], $d=0.7, p=0.43$ for $t$ test; Fig. $5 A, B)$. Gr-1 detects both Ly6G + neutrophils and Ly6C + monocytes. One day after injury, however, the majority of $\mathrm{Gr}-1+$ cells are likely to be neutrophils.

We then performed histologic assessment of immune related cells and markers of axonal damage at day 5 after $\mathrm{SCl}$. This time point was chosen because of the maintained high inflammatory reactivity of the tissue combined with the presence of macrophages in the tissue as well as signs of axonal degeneration. We quantified CD11b+ macrophages/microglia, GFAP+ astrocytes and SMI$32+$ retraction bulblike structures adjacent to the lesion epicenter. Interestingly, the amount of CD11b+ macrophages/microglia was significantly reduced in CCL3FANA ASO-treated animals, in agreement with the reduced inflammatory response $\left(M_{\text {diff }}=-1.45 e+02[95.0 \%\right.$ 
A

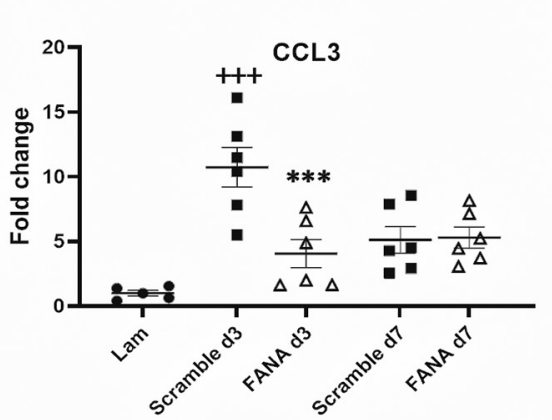

Anti-CCL3 FANA one dose

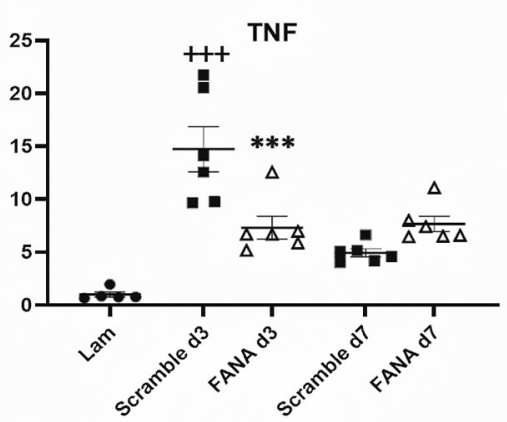

Anti-CCL3 FANA two doses

B
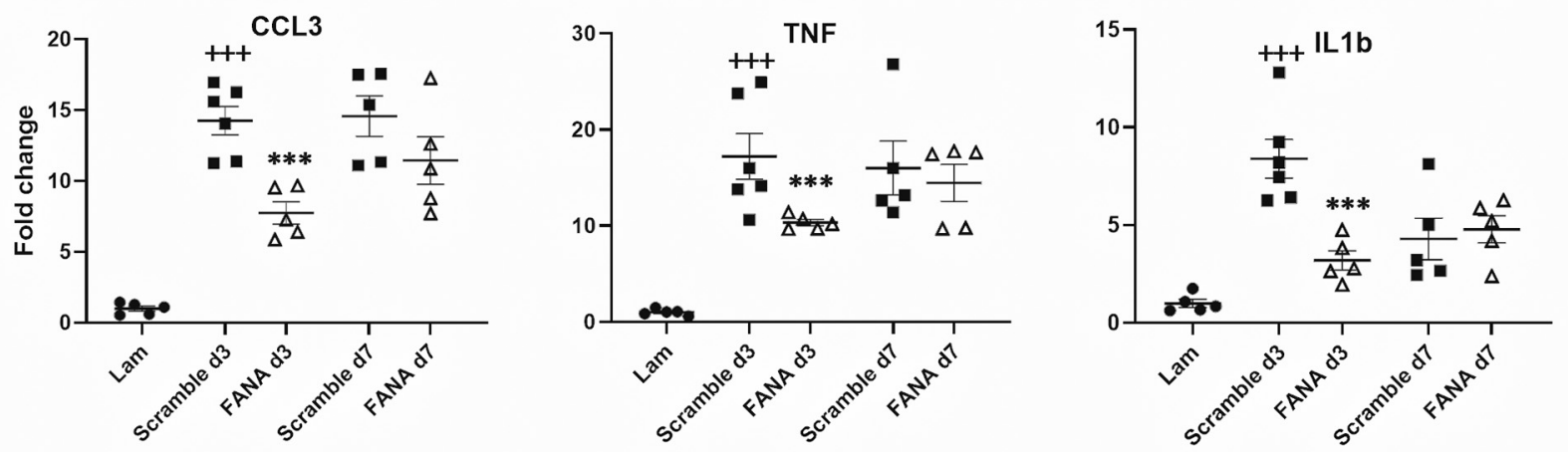

Anti-CCL3 FANA three doses

C

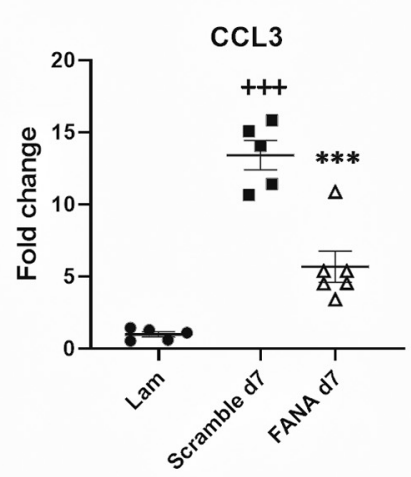

D

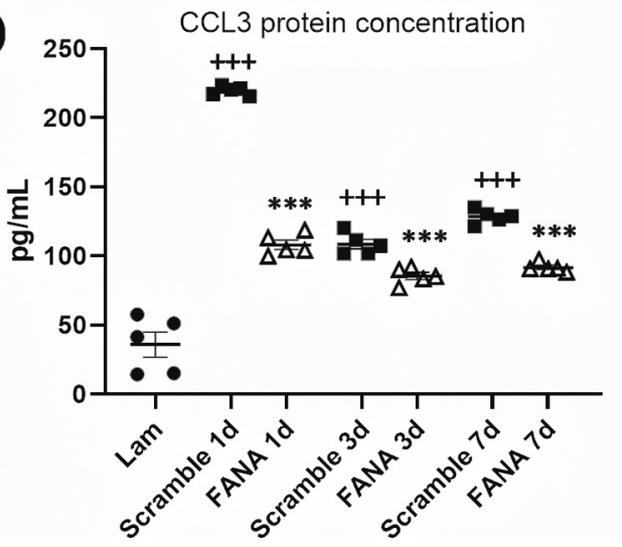

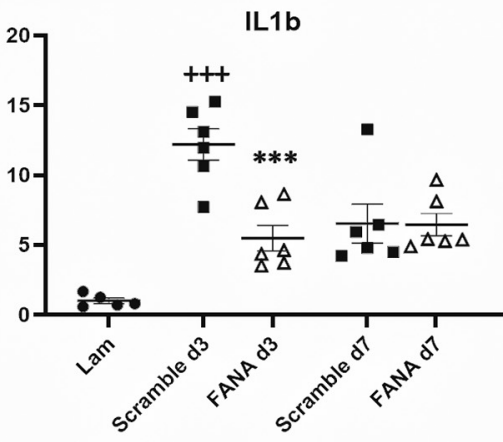

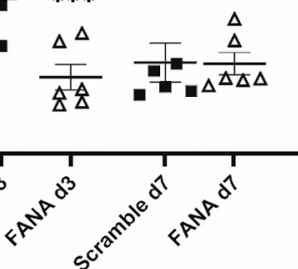
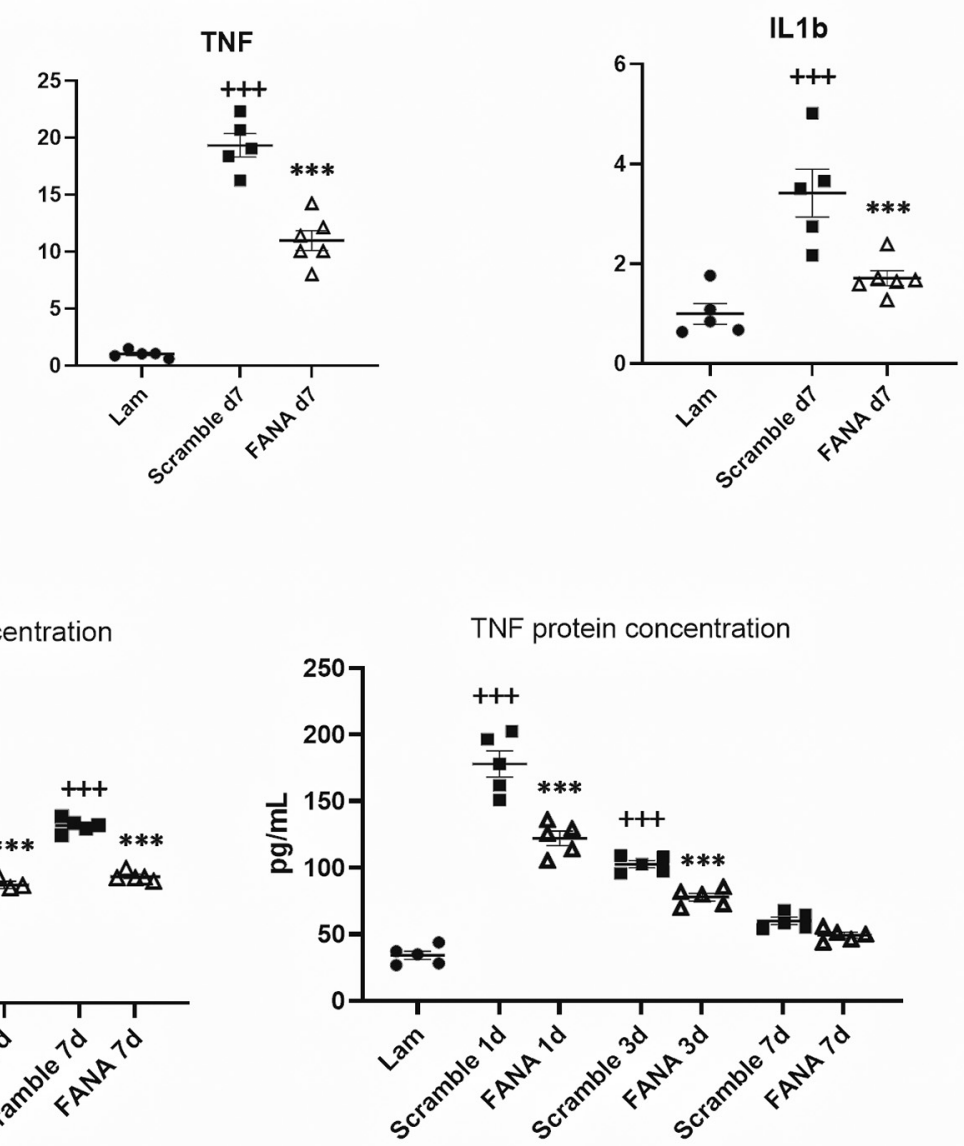
Anti-CCL3 post injury treatment
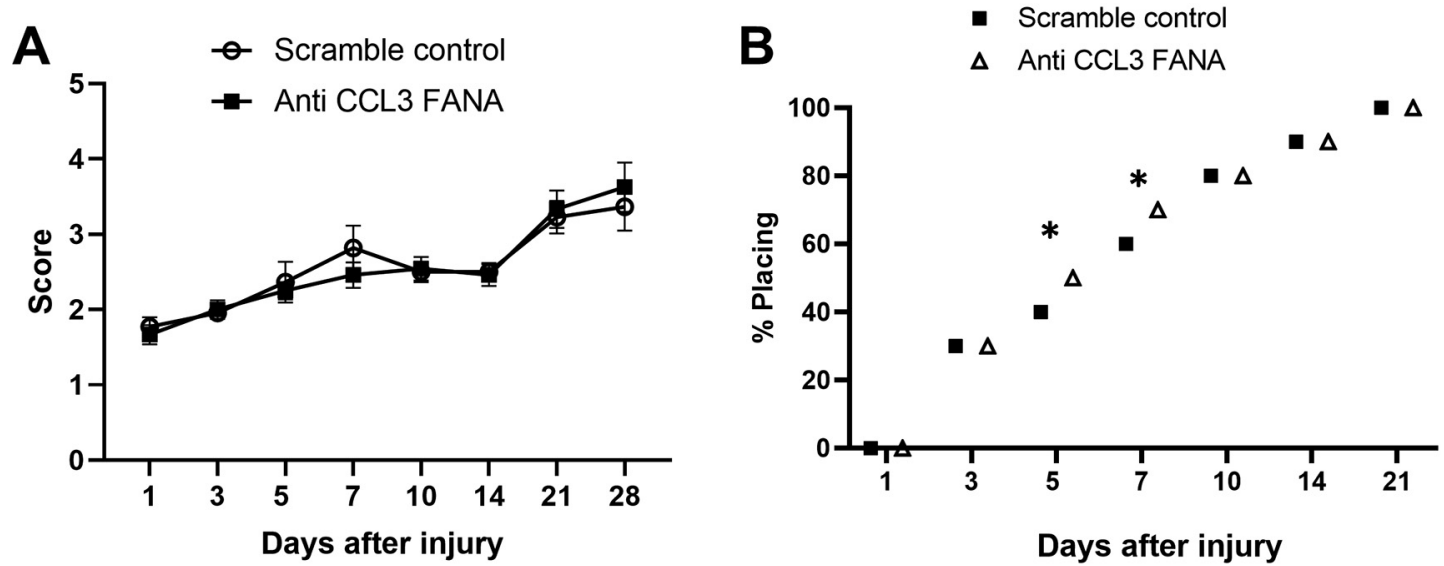

Anti-CCL3 pre injury treatment
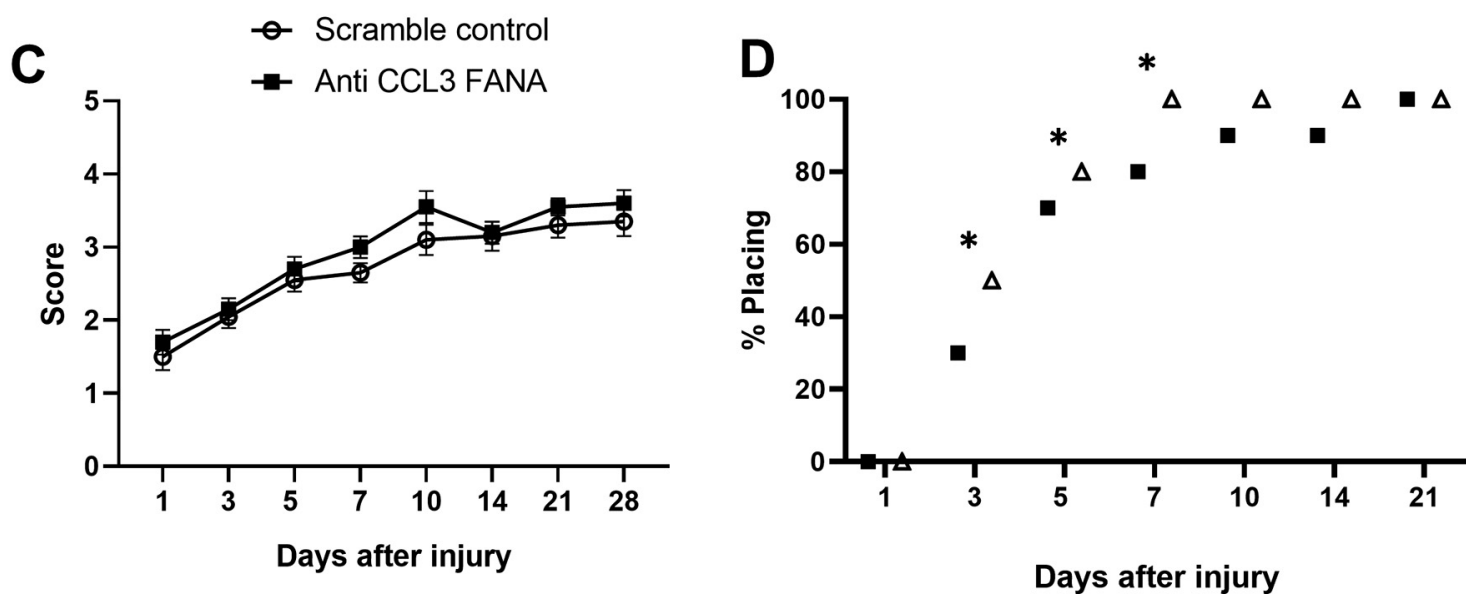

Figure 4. Treatment with anti-CCL3 FANA ASO resulted in mild functional improvement. $\boldsymbol{A}$, BMS scores did not show significant improvement compared with control animals when anti CCL3-FANA was administered three times starting right after injury. $\boldsymbol{B}$, However, a significantly higher percentage of CCL3-FANA ASO-treated mice performed plantar placement on days 5-7 after injury, compared with control animals. To ensure sufficient time to knock-down CCL3, we also investigated the efficacy of CCL3-FANA ASO pretreatment, with the first dose given $24 \mathrm{~h}$ before injury, followed by two more injection immediately after injury and $24 \mathrm{~h}$ later. $\boldsymbol{C}$, BMS scores were not significantly different in treated versus control animals when anti CCL3-FANA was administered three times starting $24 \mathrm{~h}$ before injury. $\boldsymbol{D}$. The pretreatment group, however, showed a significant increase of plantar placement at days 3 through 7 after injury compared with controls; $n=10$ /group in preinjury treatment paradigm, $n=12 /$ group in postinjury treatment. Data points are represented as mean \pm SEM. Note that bars in $\boldsymbol{B}, \boldsymbol{D}$ represent the total percentage of mice from each group capable of plantar placement, thereby not allowing for error bars; ${ }^{*} p<0.05, \chi^{2}$ test.

continued

Figure 3. CCL3 expression levels following SCI were reduced after FANA ASO injection. $\boldsymbol{A}$, A single injection of FANA ASO CCL3\#4 resulted in significant suppression of CCL3 mRNA expression in the injured spinal cord compared with scrambled control at day 3 postinjury. This reduction was accompanied by the suppression of TNF and IL-1 $\beta$ expression, indicating a reduction of the proinflammatory tissue reaction. $\boldsymbol{B}$, When using a two-injection paradigm, CCL3, TNF, and IL-1 $\beta$ mRNA were also suppressed at day 3 . C, When three doses were administered, a sustained suppression of CCL3 was detected at day 7 , reducing CCL3 levels by $\sim 60 \%$. Both TNF and IL-1 $\beta$ gene expression levels were reduced by $\sim 50 \%$. $\boldsymbol{D}$, Suppression of CCL3 and TNF in the injured spinal cord was also measured the protein level using ELISA at days 1, 3, and 7 and days 1 and 3 after SCI, respectively; $n=5 / g r o u p ;$ $+++p<0.001$ (compared with laminectomy control); ${ }^{*} p<0.05,{ }^{* * *} p<0.001$ (compared with scrambled control of the same time point). Error bars are represented as SEM. 
A

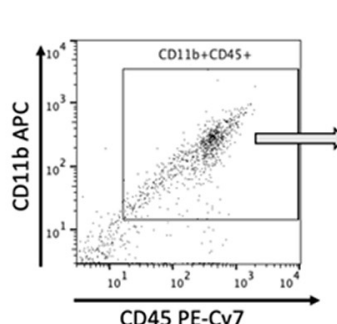

C
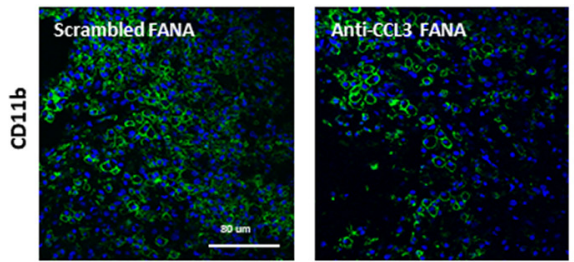

Macrophages

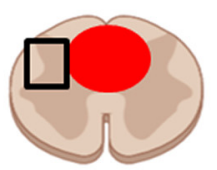

D

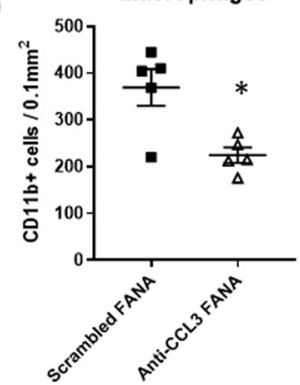

$10^{1} \quad 12^{2}$
Negative control
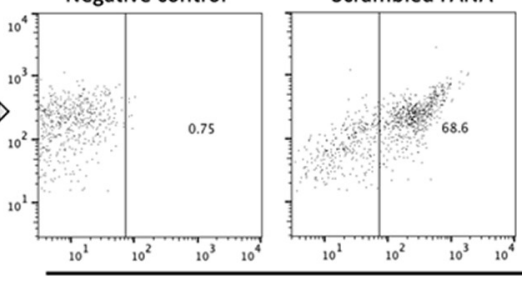

Gr-1 FITC

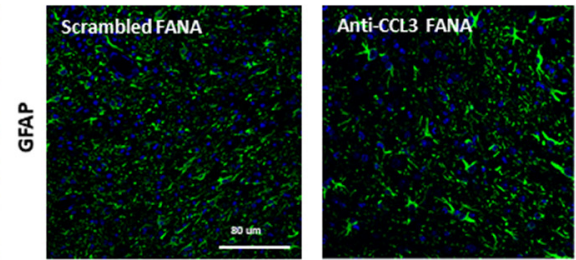

Astrocytes

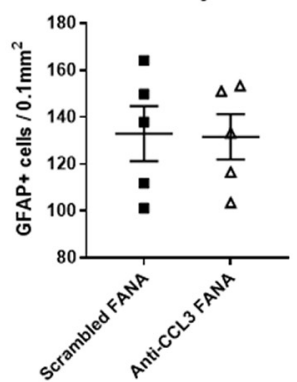

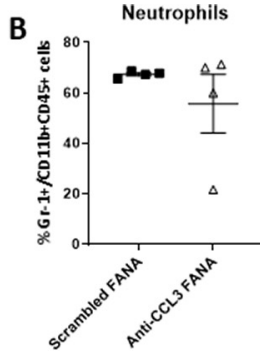
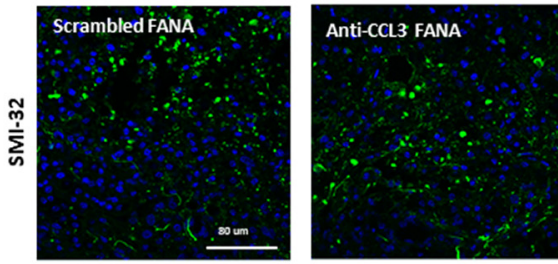

Axonal damage

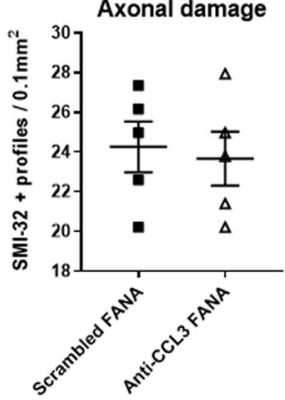

Figure 5. Treatment with anti-CCL3 FANA ASOs reduced CD11b+ macrophages at the lesion site. $A$, B. Flow cytometry $1 \mathrm{~d}$ after $\mathrm{SCl}$ in anti-CCL3-FANA ASO and control-treated animals did not reveal a significant difference in Gr-1+ neutrophils. Numbers were assessed in the CD11b $+C D 45+$ gated cell population. The negative control was stained with CD11b+CD45+ in absence of the Gr-1 antibody. A, Representative flow cytometry images. B, Quantification of CD11b+CD45+Gr-1+ neutrophils. C, Representative images of CD11b + macrophages/microglia, GFAP+ astrocytes, and SMI-32+ profiles as indicators of axonal damage adjacent to the lesion epicenter in anti-CCL3-FANA ASO and control-treated animals. The schematic indicates the location of the quantified region (square) next to the lesion (red circle). $\boldsymbol{D}$, Quantification revealed a significant reduction of CD11b+ macrophages/microglia in the anti-CCL3-FANA ASO treatment group, while neither astrocytes nor indicators of axonal damage were different between groups; $n=5 /$ group, data points are represented as mean \pm SEM; ${ }^{*} p=0.01$. Scale bars: $80 \mu \mathrm{m}$.

$\mathrm{Cl}-2 \mathrm{e}+02,-38.5], d=2.15, p=0.01$ for $t$ test). Astrocyte numbers and indicators of axonal damage (SMI-32+ profiles) were not different between groups $\left(\mathrm{M}_{\text {diff }}=-1.43[95.0 \% \mathrm{Cl}\right.$ $-27.6,24.7], d=0.06, p=0.94$ for $t$ test $)$ and $\left(M_{\text {diff }}-0.5\right.$ $[95.0 \% \mathrm{Cl}-3.2,2.4], d=0.021, p=0.72$ for $t$ test; Fig. $5 C, D$ ).

\section{CCL3 inhibition with specific FANA ASOs did not significantly reduce tissue damage after $\mathrm{SCI}$}

Finally, we quantified lesion sizes in the injured spinal cords of CCL3-FANA ASO-treated and control mice. Immunofluorescent staining for GFAP and FluoroMyelin was used to examine the lesion size as well as the amount of preserved myelin in the injured spinal cords (Fig. 6A). Quantitative analysis of the lesion volume did not show a significant reduction in lesion size in CCL3-FANA ASOpretreated mice (Fig. 6B). No differences in myelin content, as shown with FluoroMyelin Red staining, were detected between groups (Fig. 6C).

\section{Discussion}

Uncontrolled inflammation plays a critical role during secondary tissue damage following traumatic SCl. In particular, proinflammatory cytokines and chemokines have been shown to play a main role in exacerbating the secondary damage after the primary injury. Specifically targeting proinflammatory molecules in the injured spinal cord can present technical challenges in regard to tissue penetration, stability and efficacy of suppression. The use of specific FANA ASO molecules might be a feasible approach to circumvent these issues. Here, we set out to determine the efficacy of this innovative strategy to specifically target inflammatory molecules after SCI. Taken together, our experiments demonstrate both the feasibility in using FANA ASO molecules after SCl and their anti-inflammatory potential in the injured tissue.

Our results indicate that intrathecal administration of anti-CCL3 FANA ASO treatment provides a significant downregulation of CCL3 and others proinflammatory cytokines. This is of interest because it demonstrates the local efficacy of CCL3 suppression as well as the impact on the inflammatory tissue environment. Moreover, targeting CCL3 using this technology led to a slight improvement of locomotor recovery. These results support our previous data showing the role of CCL3 in modulating the inflammatory response and secondary degeneration after 

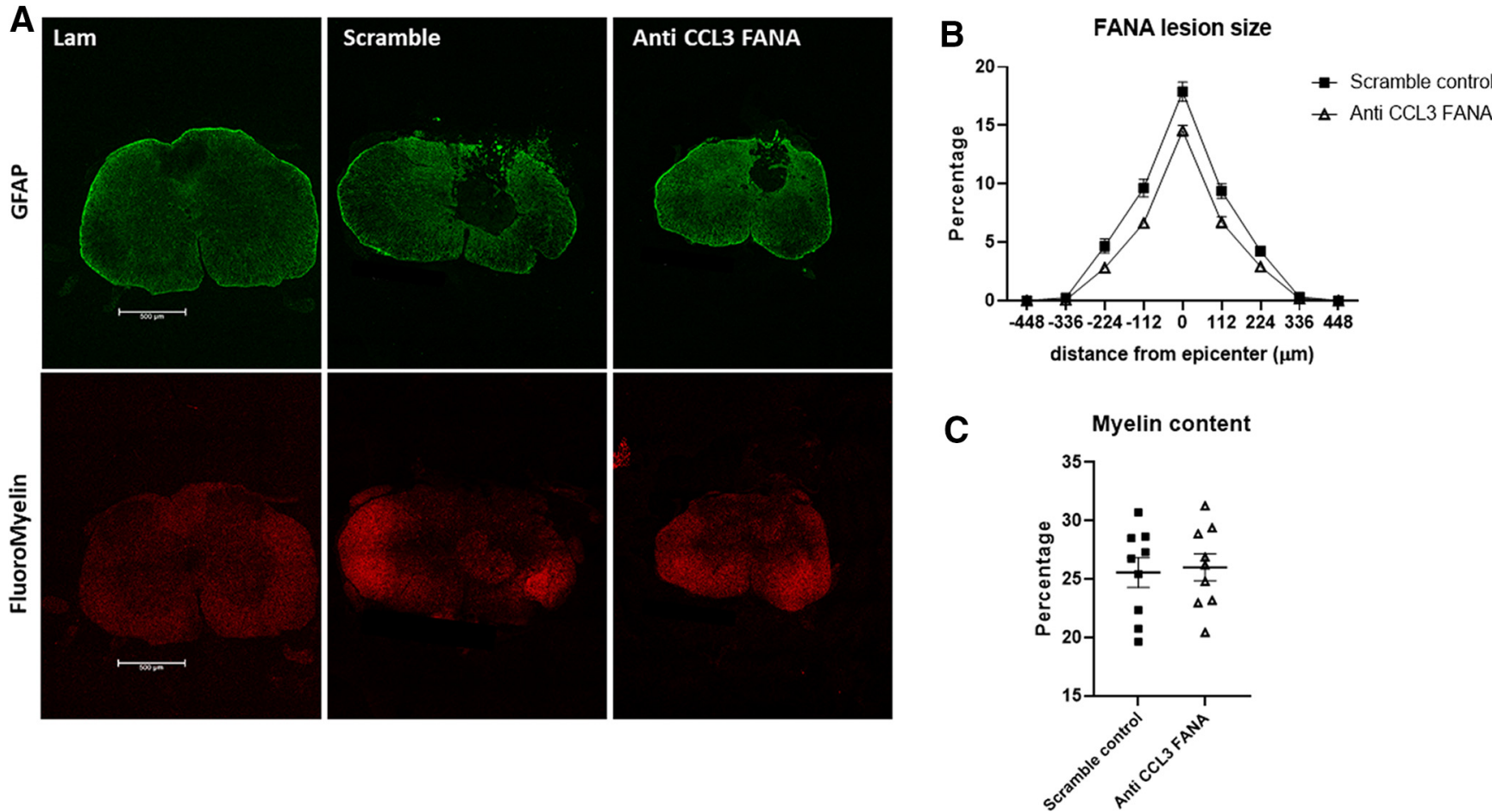

Figure 6. CCL3 inhibition with FANA ASOs did not significantly reduce tissue damage after SCI. Quantification of areas devoid of GFAP immunoreactivity at day 28 after SCI did not show a significant difference in lesion area in anti-CCL3-FANA ASO-pretreated mice compared with control mice. $\boldsymbol{A}$, Representative images of GFAP and FluoroMyelin stained spinal cord cross sections. $\boldsymbol{B}$, Quantitative analysis of the lesion volume in CCL3-FANA ASO-pretreated mice and control mice. $\boldsymbol{C}$, No differences in myelin content, as shown with FluoroMyelin Red staining, were detected between groups; $n=9 /$ group. Data points are represented as mean \pm SEM. Scale bars: $500 \mu \mathrm{m}$.

$\mathrm{SCl}$. This warrants further studies to examine this technology to target CCL3 and other inflammatory molecules for reducing secondary damage and improving functional recovery.

In the past years, ASOs have been validated as therapeutic agents to generate mRNA selective knock-downs both in vitro and in vivo. However, successful application was limited by critical technical issues. These issues included insufficient stability of RNA inhibitors because of nucleases degradation activity, and poor cellular delivery as a result of low cellular uptake from inefficient crossing of cellular membranes. For this, next generation ASOs, such as FANA ASO molecules, have been developed to improve nuclease resistance, increase binding affinity, and to enhance biostability. One of the most important modification to the biological function of FANA ASO molecules is their ability to penetrate cells without the necessity of delivery vehicles required in transfection approaches. Our results revealed that FANA ASO molecules targeting CCL3 can indeed penetrate BMDMs in gymnotic fashion, as previously described for other cell types in vitro (Schmidt et al., 2019; Smaldone et al., 2019; Suwanmanee et al., 2019; Takahashi et al., 2019; Baby et al., 2020; Margiotta and Howard, 2020). We also demonstrated the successful suppression of CCL3 expression in BMDMs by CCL3 specific FANA ASOs. Out of four molecules targeting different areas of the CCL3 mRNA that we investigated, one molecule mediated significantly reduced CCL3 expression, while three others did not significantly suppress CCL 3 in vitro.
As a next important step, we assessed the feasibility of in vivo application of these FANA ASO molecules after SCI and in uninjured animals. We observed gymnotic tissue penetration after intrathecal injection, when fluorescently labeled FANA ASO molecules were detected in the spinal cord, primarily at the lesion site. Using flow cytometry, FANA uptake could be detected in CD11b+CD45low microglia (MG) of naive mice and $7 \mathrm{~d}$ postinjury mice. SCI mice also showed labeling of CD11b+CD45high MDMs, a cell population which, as expected, are not present in naive animals. CD11b is a myeloid cell marker commonly used to identify MG and MDM in combination with CD45 expression levels. Both CD11b and CD45 are also expressed on neutrophils. However, at this time point after $\mathrm{SCl}$, the majority of myeloid cells in the tissue are expected to be microglia or MDM.

The percentage of labeled cells showed a high variability within the group, which may be explained by less successful intrathecal injections. Further work will have to be done to optimize administration and availability of FANA ASO molecules in the injured spinal cord.

Fluorescently labeled FANA ASO could also be detected histologically, with the highest concentrations being close to the lesion site. Its uptake was not cell specific, as we observed FANA ASO positive macrophages/ microglia and astrocytes. Interestingly, no penetration was detected in NeuN+ neurons, which could be explained by their localization with some distance to the lesion epicenter. 
When assessing the efficacy of CCL3 suppression after intrathecal injection, we achieved a gene expression reduction of $50-60 \%$ in the injured tissue $3 \mathrm{~d}$ after SCl. This suppression level was comparable when one or two doses of FANA ASO were administered intrathecally. However, neither one nor two doses of FANA ASO, immediately and $24 \mathrm{~h}$ after injury, were sufficient to significantly suppress CCL3 expression levels persistently for $7 d$ after $\mathrm{SCl}$. When a third dose was added $48 \mathrm{~h}$ following injury, CCL3 expression levels were significantly reduced by $62 \%$ in the injured tissue $7 \mathrm{~d}$ after injury. This significant reduction of CCL3 after three doses was confirmed on the protein level using ELISA. FANA ASO mediated mRNA suppression was observed to the same extent in a model of Huntington's disease, where the suppression persisted for 12 weeks after continuous infusion for two weeks (Kordasiewicz et al., 2012). In vitro, sustained antisense activity has been described for other FANA ASO molecules for four to $13 \mathrm{~d}$ (Ferrari et al., 2006; Takahashi et al., 2019).

Interestingly, the levels of the proinflammatory cytokines TNF and IL-1 $\beta$ closely resembled the levels of CCL3 after different administration paradigms. TNF concentration was confirmed on the protein levels to be reduced at days 1 and 3 after $\mathrm{SCl}$. No significant reduction was detectable at day 7 . At this time, overall TNF levels had returned to baseline expression. We could also demonstrate that the treatment with anti-CCL3 FANA ASO reduced the number of macrophages adjacent to the lesion $5 \mathrm{~d}$ after injury, a finding that closely resembles our observation in $\mathrm{CCL3}^{-1-}$ mice (Pelisch et al., 2020).

These findings support the importance of CCL3 as an inflammatory chemokine, which regulates the production of proinflammatory cytokines and the chemotactic mobilization of immune cells into inflammatory tissues (Baba and Mukaida, 2014). It is important to consider that all experiments were performed in 8- to 10-week-old mice. While this age group is frequently used in $\mathrm{SCl}$ research, it has been described that the inflammatory response after $\mathrm{SCl}$ in young mice is less pronounced compared with older mice (Zhang et al., 2015). The use of different age groups may reveal different effects on the inflammatory response in future experiments.

After demonstrating the efficacy of FANA ASO molecules to reduce CCL3 expression following SCl in vivo, we assessed the impact on functional recovery. Open field locomotor (BMS) score showed no significant differences in mice receiving anti-CCL3 FANA ASO treatment compared with scrambled control. However, a significantly higher percentage of mice treated with CCL3-FANA ASO performed plantar placement on days 5-7 after injury, compared with control animals, confirming that CCL3 knockdown before and/or after injury with CCL3-FANA ASO can result in a mild functional improvement. However, treatment with FANA ASO did not result in a significant reduction of lesion size. Furthermore, no difference in axonal damage, assessed by quantification of SMI-32+ profiles, was detected at day 5 after injury. These results are in line with the absence of preserved behavioral improvement at later time points after injury. The absence of more pronounced behavioral differences or tissue preservation could be explained by the level of CCL3 suppression. Complete absence of CCL3 in CCL3 deficient mice results in a slightly but significantly reduced functional recovery and reduction of lesion sizes (our unpublished data). However, the suppression of CCL3 in our SCI model reached $\sim 60 \%$, which could lead to a reduced functional impact.

Our previous work in $\mathrm{CCL}^{-/-}$mice demonstrated improved locomotor recovery and reduced tissue damage. It is therefore tempting to speculate that increased efficacy of suppression would result in more pronounced tissue preservation and functional recovery.

In summary, for the first time, this study demonstrates the feasibility and potential of the use of FANA ASO molecules in vivo targeting specific molecules in the injured or pathologically altered spinal cord tissue. The use of this technology could provide important treatment options to mitigate secondary damage following $\mathrm{SCl}$ as well other diseases of the brain and spinal cord. Moreover, we demonstrate that targeting CCL3 using a novel mRNA inhibitor, FANA ASO, results in a reduced proinflammatory response followed by a mild but significant functional improvement after $\mathrm{SCl}$.

\section{References}

Baba T, Mukaida N (2014) Role of macrophage inflammatory protein (MIP)-1 $\alpha$ /CCL3 in leukemogenesis. Mol Cell Oncol 1:e29899.

Baby N, Alagappan N, Dheen ST, Sajikumar S (2020) MicroRNA134-5p inhibition rescues long-term plasticity and synaptic tagging/capture in an $A \beta(1-42)$-induced model of Alzheimer's disease. Aging Cell 19:e13046.

Banisor I, Leist TP, Kalman B (2005) Involvement of beta-chemokines in the development of inflammatory demyelination. $J$ Neuroinflammation 2:7.

Bartholdi D, Schwab M (1997) Expression of pro-inflammatory cytokine and chemokine mRNA upon experimental spinal cord injury in mouse: an in situ hybridization study. Eur J Neurosci 9:1422-1438.

Basso DM, Fisher LC, Anderson AJ, Jakeman LB, McTigue DM, Popovich PG (2006) Basso Mouse Scale for locomotion detects differences in recovery after spinal cord injury in five common mouse strains. J Neurotrauma 23:635-659.

Bennett CF, Swayze EE (2010) RNA targeting therapeutics: molecular mechanisms of antisense oligonucleotides as a therapeutic platform. Annu Rev Pharmacol Toxicol 50:259-293.

Bhagavati S (2016) Doubts about therapy for neurological diseases with antisense oligonucleotides. JAMA Neurol 73:1502.

Blight AR (1994) Effects of silica on the outcome from experimental spinal cord injury: implication of macrophages in secondary tissue damage. Neuroscience 60:263-273.

Brennan FH, Lee JD, Ruitenberg MJ, Woodruff TM (2016) Therapeutic targeting of complement to modify disease course and improve outcomes in neurological conditions. Semin Immunol 28:292-308.

Chi X, Gatti P, Papoian T (2017) Safety of antisense oligonucleotide and siRNA-based therapeutics. Drug Discov Today 22:823-833.

Chorzalska A, Morgan J, Ahsan N, Treaba DO, Olszewski AJ, Petersen M, Kingston N, Cheng Y, Lombardo K, Schorl C, Yu X, Zini R, Pacilli A, Tepper A, Coburn J, Hryniewicz-Jankowska A, Zhao TC, Oancea E, Reagan JL, Liang O, et al. (2018) Bone marrow-specific loss of $\mathrm{ABI}$ induces myeloproliferative neoplasm with features resembling human myelofibrosis. Blood 132:20532066. 
Denisov AY, Noronha AM, Wilds CJ, Trempe JF, Pon RT, Gehring K, Damha MJ (2001) Solution structure of an arabinonucleic acid (ANA)/RNA duplex in a chimeric hairpin: comparison with 2'-fluoro-ANA/RNA and DNA/RNA hybrids. Nucleic Acids Res 29:42844293.

Donnelly DJ, Popovich PG (2008) Inflammation and its role in neuroprotection, axonal regeneration and functional recovery after spinal cord injury. Exp Neurol 209:378-388.

Ferrari N, Bergeron D, Tedeschi AL, Mangos MM, Paquet L, Renzi PM, Damha MJ (2006) Characterization of antisense oligonucleotides comprising 2'-deoxy-2'-fluoro-beta-D-arabinonucleic acid (FANA): specificity, potency, and duration of activity. Ann NY Acad Sci 1082:91-102.

Fortin M, D'Anjou H, Higgins ME, Gougeon J, Aubé P, Moktefi K, Mouissi S, Séguin S, Séguin R, Renzi PM, Paquet L, Ferrari N (2009) A multi-target antisense approach against PDE4 and PDE7 reduces smoke-induced lung inflammation in mice. Respir Res 10:39.

Francos-Quijorna I, Santos-Nogueira E, Gronert K, Sullivan AB, Kopp MA, Brommer B, David S, Schwab JM, López-Vales R (2017) Maresin 1 promotes inflammatory resolution, neuroprotection, and functional neurological recovery after spinal cord injury. $J$ Neurosci 37:11731-11743.

Gustincich S, Zucchelli S, Mallamaci A (2017) The Yin and Yang of nucleic acid-based therapy in the brain. Prog Neurobiol 155:194211.

Ho J, Tumkaya T, Aryal S, Choi H, Claridge-Chang A (2019) Moving beyond $P$ values: data analysis with estimation graphics. Nat Methods 16:565-566.

Kasama T, Strieter RM, Standiford TJ, Burdick MD, Kunkel SL (1993) Expression and regulation of human neutrophil-derived macrophage inflammatory protein 1 alpha. J Exp Med 178:63-72.

Kordasiewicz HB, Stanek LM, Wancewicz EV, Mazur C, McAlonis MM, Pytel KA, Artates JW, Weiss A, Cheng SH, Shihabuddin LS, Hung G, Bennett CF, Cleveland DW (2012) Sustained therapeutic reversal of Huntington's disease by transient repression of huntingtin synthesis. Neuron 74:1031-1044.

Kroner A, Greenhalgh AD, Zarruk JG, Passos Dos Santos R, Gaestel M, David S (2014) TNF and increased intracellular iron alter macrophage polarization to a detrimental M1 phenotype in the injured spinal cord. Neuron 83:1098-1116.

Lee YL, Shih K, Bao P, Ghirnikar RS, Eng LF (2000) Cytokine chemokine expression in contused rat spinal cord. Neurochem Int 36:417-425.

Lok CN, Viazovkina E, Min KL, Nagy E, Wilds CJ, Damha MJ, Parniak MA (2002) Potent gene-specific inhibitory properties of mixedbackbone antisense oligonucleotides comprised of 2'-deoxy-2'fluoro-D-arabinose and 2'-deoxyribose nucleotides. Biochemistry 41:3457-3467.

Longbrake EE, Lai W, Ankeny DP, Popovich PG (2007) Characterization and modeling of monocyte-derived macrophages after spinal cord injury. J Neurochem 102:1083-1094.

Margiotta JF, Howard MJ (2020) Cryptochromes mediate intrinsic photomechanical transduction in avian iris and somatic striated muscle. Front Physiol 11:128.

Martín-Pintado N, Yahyaee-Anzahaee M, Campos-Olivas R, Noronha AM, Wilds CJ, Damha MJ, González C (2012) The solution structure of double helical arabino nucleic acids (ANA and 2'F-ANA): effect of arabinoses in duplex-hairpin interconversion. Nucleic Acids Res 40:9329-9339.

Min KL, Viazovkina E, Galarneau A, Parniak MA, Damha MJ (2002) Oligonucleotides comprised of alternating 2'-deoxy-2'-fluoro-
$\beta$-D-arabinonucleosides and D-2'-deoxyribonucleosides (2'FANA/DNA 'altimers') induce efficient RNA cleavage mediated by RNase H. Bioorg Med Chem Lett 12:2651-2654.

Njoo C, Heinl C, Kuner R (2014) In vivo SiRNA transfection and gene knockdown in spinal cord via rapid noninvasive lumbar intrathecal injections in mice. J Vis Exp. Advance online publication. Retrieved Mar 22, 2014. doi: 10.3791/51229.

Østergaard ME, Southwell AL, Kordasiewicz H, Watt AT, Skotte NH, Doty CN, Vaid K, Villanueva EB, Swayze EE, Bennett CF, Hayden MR, Seth PP (2013) Rational design of antisense oligonucleotides targeting single nucleotide polymorphisms for potent and allele selective suppression of mutant Huntingtin in the CNS. Nucleic Acids Res 41:9634-9650.

Pelisch N, Rosas Almanza J, Stehlik KE, Aperi BV, Kroner A (2020) CCL3 contributes to secondary damage after spinal cord injury. $J$ Neuroinflammation 17:362.

Peterson PK, Hu S, Salak-Johnson J, Molitor TW, Chao CC (1997) Differential production of and migratory response to beta chemokines by human microglia and astrocytes. J Infect Dis 175:478481.

Sato A, Ohtaki H, Tsumuraya T, Song D, Ohara K, Asano M, Iwakura Y, Atsumi T, Shioda S (2012) Interleukin-1 participates in the classical and alternative activation of microglia/macrophages after spinal cord injury. J Neuroinflammation 9:65.

Schmidt K, Carroll JS, Yee E, Thomas DD, Wert-Lamas L, Neier SC, Sheynkman G, Ritz J, Novina CD (2019) The IncRNA SLNCR recruits the androgen receptor to EGR1-bound genes in melanoma and inhibits expression of tumor suppressor p21. Cell Rep 27:2493-2507.e4.

Sekhon LH, Fehlings MG (2001) Epidemiology, demographics, and pathophysiology of acute spinal cord injury. Spine (Phila Pa 1976) 26:S2-S12.

Singh A, Tetreault L, Kalsi-Ryan S, Nouri A, Fehlings MG (2014) Global prevalence and incidence of traumatic spinal cord injury. Clin Epidemiol 6:309-331.

Smaldone $G$, Beneduce $G$, Incoronato $M$, Pane K, Franzese $M$, Coppola L, Cordella A, Parasole R, Ripaldi M, Nassa G, Soricelli A, Vitagliano L, Mirabelli P, Salvatore M (2019) KCTD15 is overexpressed in human childhood B-cell acute lymphoid leukemia. Sci Rep 9:20108.

Sun D, Hu X, Liu X, Whitaker JN, Walker WS (1997) Expression of chemokine genes in rat glial cells: the effect of myelin basic protein-reactive encephalitogenic T cells. J Neurosci Res 48:192-200.

Suwanmanee S, Mahakhunkijcharoen Y, Ampawong S, Leaungwutiwong P, Missé D, Luplertlop N (2019) Inhibition of N-myristoyltransferase1 affects dengue virus replication. Microbiologyopen 8:e00831.

Takahashi M, Li H, Zhou J, Chomchan P, Aishwarya V, Damha MJ, Rossi JJ (2019) Dual mechanisms of action of self-delivering, antiHIV-1 FANA oligonucleotides as a potential new approach to HIV therapy. Mol Ther Nucleic Acids 17:615-625.

Standiford TJ, Kunkel SL, Liebler JM, Burdick MD, Gilbert AR, Strieter RM (1993) Gene expression of macrophage inflammatory protein-1 $\alpha$ from human blood monocytes and alveolar macrophages is inhibited by interleukin-4. Am J Respir Cell Mol Biol 9:192-198.

Zhang B, Bailey WM, Braun KJ, Gensel JC (2015) Age decreases macrophage IL-10 expression: implications for functional recovery and tissue repair in spinal cord injury. Exp Neurol 273:83-91.

Zhang G, Yang P (2017) Bioinformatics genes and pathway analysis for chronic neuropathic pain after spinal cord injury. Biomed Res Int 2017:6423021. 